\title{
Induction of Engineered Residual Stresses Fields and Associate Surface Properties Modification by Short Pulse Laser Shock Processing
}

\author{
J.L. Ocaña ${ }^{1}$, M. Morales ${ }^{1}$, J.A. Porro', M. Blasco ${ }^{1}$, C. Molpeceres ${ }^{1}$, D. Iordachescu ${ }^{1}$, \\ G. Gómez-Rosas ${ }^{2}$, C. Rubio-González ${ }^{3}$ \\ ${ }^{1}$ Centro Láser UPM. Universidad Politécnica de Madrid. \\ Campus Sur UPM. Edificio La Arboleda. Ctra. de Valencia, km. 7,300. 28031 Madrid. SPAIN. \\ Tel.: (+34) 913363099. Fax: (+34) 913365534. email: jlocana@etsii.upm.es \\ ${ }^{2}$ CUCEI. Universidad de Guadalajara. Guadalajara. Jalisco. MEXICO \\ ${ }^{3}$ CIDESI. Pie de la Cuesta, 702. Desarrollo San Pablo. Santiago de Querétaro. Qro. MEXICO
}

Keywords: Laser shock processing, thermo-mechanical effects, residual stresses, surface
properties, roughness, hardness, wear, fatigue

\begin{abstract}
Laser shock processing (LSP) is consolidating as an effective technology for the improvement of metallic materials surface properties involving their fatigue life. The main acknowledged advantage of the LSP technique consists on its capability of inducing a relatively deep compression residual stresses field into metallic alloy pieces allowing an improved mechanical behaviour, explicitly the life improvement of the treated specimens against wear, crack growth and stress corrosion cracking. Progress accomplished by the authors in the line of practical development of the LSP technique at an experimental level, aiming its integral assessment from an interrelated theoretical and experimental point of view, is presented in this paper. Concretely, experimental results on the residual stress profiles and associated surface properties modification successfully reached in typical materials (especially $\mathrm{Al}$ and $\mathrm{Ti}$ alloys) under different LSP irradiation conditions are presented, a correlated analysis of the residual stress profiles obtained under different irradiation strategies and the evaluation of the corresponding induced surface properties as roughness and wear resistance being also presented. Through a coupled theoretical- experimental analysis the real possibilities of the LSP technique as a possible substitutive of related traditional surface modification techniques as, for example, shot peening.
\end{abstract}

\section{Introduction}

Laser shock processing is consolidating as a competitive alternative technology to classical treatments for the improvement of surface properties of metallic alloys involving the fatigue life of critical components. Specially wear resistance, stress corrosion cracking susceptibility and crack propagation rate seem to be material properties specially improved by LSP treatments [1-4].

The technique was initially developed for the improvement of the fatigue cracking resistance of materials used in the aeronautic applications. Materials such as Aluminum and Titanium alloys and different types of stainless steel were extensively investigated but the unavailability of laser sources able to provide the required high intensities prevented a wide industrial implementation of the technique. More recently, on the basis of the commercial availability of new powerful laser sources able to provide intensities exceeding the $\mathrm{GW} / \mathrm{cm}^{2}$ level, a new intense research effort aims to develop the LSP technology from an industrial point of view [5-8]. The authors have made previous contributions to the field showing initial results of their developments [9-17].

In this paper, new experimental results on the residual stress profiles created in $\mathrm{Al}$ and $\mathrm{Ti}$ alloys under different irradiation conditions are presented along with the associate effects on characteristic material surface properties, namely surface roughness and wear resistance. Based on the analysis of these results, experimental guidelines are obtained allowing a critical assessment of the LSP technique as potentially competitive to traditional related techniques as shot peening. 


\section{Experimental setup}

The practical irradiation system used for the experiments reported in this paper is schematically and photographically shown in Fig. 1. Using purified water as confining medium, the test piece is fixed on a holder and is driven along $\mathrm{X}$ and $\mathrm{Y}$ directions by means of a computer controlled stage needed for the irradiation of extended areas of material following a pre-defined pulse overlapping strategy.

The laser light is then conducted to the interaction area by means of a reflecting mirror and a focusing lens. The control of the purity of the confining medium is important in order to avoid the formation of water bubbles or increasing concentration of impurities resulting from material ablation following the laser irradiation.
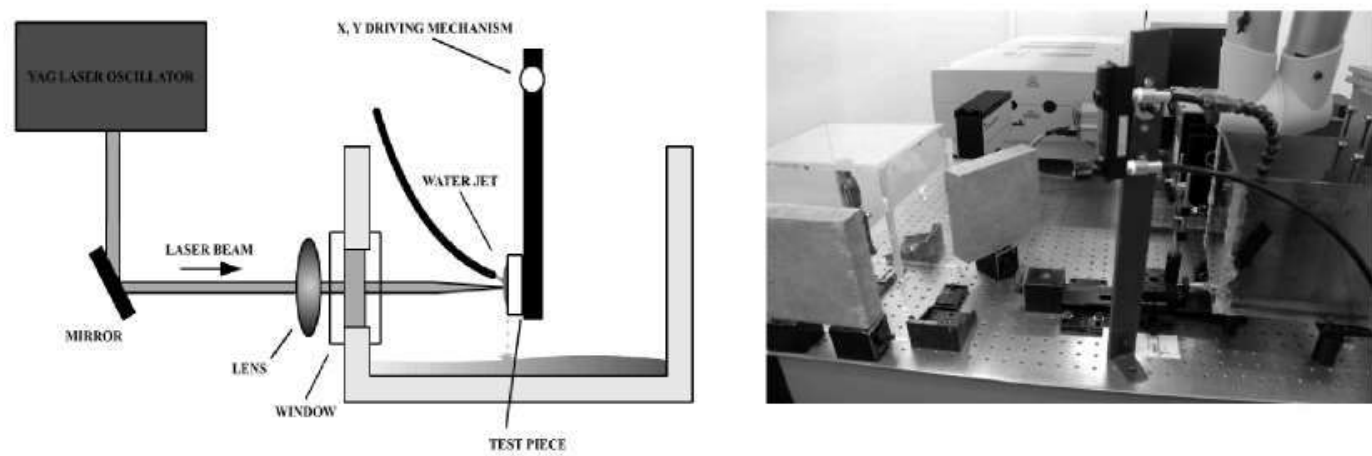

Figure 1: Schematic representation and photographic view of the LSP irradiation experimental setup used in experiments.

The LSP experiments reported in this paper were performed on A12024-T351 and Ti6Al4V alloys at $1064 \mathrm{~nm}$ laser wavelength using a Q switched $\mathrm{Nd}$ :YAG laser operating at $10 \mathrm{~Hz}$ and providing $9.4 \mathrm{~ns}$ FWHM, 2.0 J pulses. A convergent lens was used to deliver the laser energy over a $1.5 \mathrm{~mm}$ spot diameter. The confining layer was provided by a water jet incident close to the laser interaction zone. No protective coatings were used in the experiments.

The test piece geometry used for the investigations is displayed in Fig. 2 together with a photograph of the resulting aspect of the work piece after the application of the LSP treatment and subsequent residual stresses field determination by the hole drilling method.
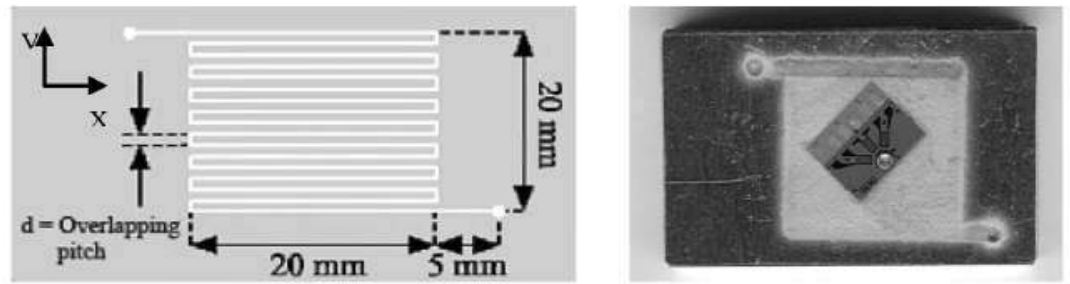

Figure 2. Schematic representation of the LSP surface sweeping strategy and photograph showing a real A12024-T351 test piece after processing.

In this test piece, the varying experimental parameter is the so-called "overlapping pitch", $d$, a direct measure of the distance between both adjacent laser shots and parallel processing tracks which, in the defined geometry, implies a given pulse density according to the relation:

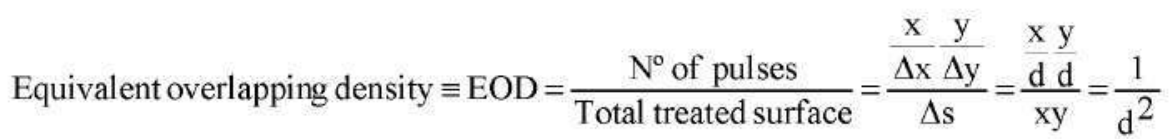

as well as an equivalent local overlapping factor given by the relation:

$$
\underset{\text { overlapping factor }}{\text { Equivalentlocal }} \equiv \mathrm{ELOF}=\begin{gathered}
\text { Averagenumber of pulses } \\
\text { at a given location }
\end{gathered}=\frac{\mathrm{N}^{\circ} \text { of pulses } \mathrm{x} \text { Area of single pulse }}{\text { Total treated surface }}=\frac{\frac{\pi}{4} \phi^{2}}{\mathrm{~d}^{2}}=\frac{\pi}{4}\left(\frac{\phi}{\mathrm{d}}\right)^{2}
$$




\section{Experimental results}

Under the referred experimental conditions, the effects induced by the LSP treatment on the mechanical properties of two characteristic alloys, namely Al2024-T351 and Ti6Al4V, have been characterized. In particular, the analysis of induced residual stress fields has been accomplished according to their primary significance. Additionally, associated surface roughness and wear resistance have been evaluated. Specimens $8 \mathrm{~mm}$ thick were used for the experiments. Equivalent overlapping densities reported in this paper were, respectively, 900,1600 and $2500 \mathrm{pulses} / \mathrm{cm}^{2}$ for A12024-T351 samples and 900, 2500 and 5000 pulses $/ \mathrm{cm}^{2}$ for Ti6Al4V samples.

Residual stress distributions were determined according to the ASTM E837-01 Standard Test Method for Determining Residual Stresses by the Hole Drilling Strain Gage Method [18]. Strain gage rosettes CEA-13-062UM-120 along with a Vishay Measurements ${ }^{\circledR}$ RS-200 milling guide were used. On its side, the wear resistance tests were performed according to the ASTM G99-04 Standard Test Method for Wear Testing with a Pin on Disk Apparatus [19] on a Microtest ${ }^{\circledR}$ MT/30 system. The samples' roughness was characterized by means of a Laser Confocal Microscope Leica ${ }^{\circledR}$ ICM 1000.

\section{Residual Stress Fields}

Fig. 3 shows the depth profiles obtained in the two materials considered for LSP-induced Mohr maximum (minimum in absolute value) residual stresses for three representative values of effective pulse density in each case.
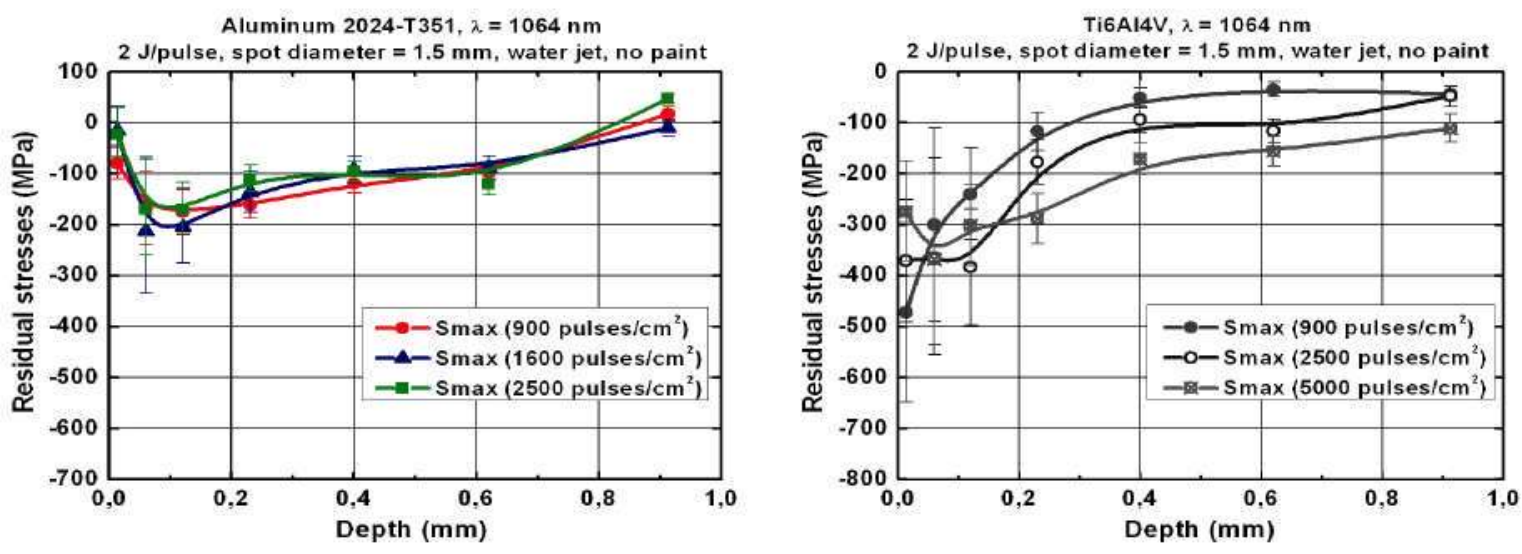

Figure 3. Comparative analysis of induced Mohr maximum residual stresses profiles along z-coordinate in A12024-T351 and Ti6Al4V for three representative pulse densities.

In both cases, the effective induction of compressive residual stress fields by means of the LSP treatment is observed, so that, in practice, in the optimum cases and taking into account that the reported values are minimum compressive stresses in absolute value, an effective protective field against crack aperture and propagation in depths up to near a millimetre can be assured.

Taking into account the respective values of Yield Strength of each of the considered materials $\left(\mathrm{YS}_{\mathrm{Al} 2024}=290 \mathrm{MPa}, \mathrm{YS}_{\mathrm{Ti} 6 \mathrm{Al} 4 \mathrm{~V}}=880 \mathrm{MPa}\right)$, it can be observed that relatively important fractional values of pre-constraint can be reached in both materials over relatively wide depths, a characteristic feature of LSP treatments that justifies its competitive character with other related existing surface treatment technologies, namely shot peening.

\section{Surface Compactation and Roughness}

In addition to residual stresses fields, surface roughness has been identified as having a significant influence on the degradation and fatigue behaviour of several critical components, especially due to corrosion and fretting fatigue [20-21].

A typical consequence of the application of the LSP technique at high ELOF's is the generation on the treated surface of roughness patterns typically smoother than those of the original workpiece, a feature that has to be considered as highly favourable in comparison with the corresponding 
results obtained by surface treatments based on thermal processing or other mechanical treatments with comparatively lower intensity and overlapping factors, such as, typically, shot peening.

In Fig. 4, a sample is provided of the surface roughness profiles (obtained via Laser Confocal Microscopy) induced by the LSP technique on the two materials considered in the study for nearlyoptimum EOD's. In both cases a clear improvement of the material surface roughness can be appreciated in comparison with the untreated material, this modification being accompanied in the case of Al2024-T351 (with a lower elastic limit) of a significant plastic deformation. In Table 1, the corresponding values of the plastically induced surface level compactation, $\langle\Delta \mathbf{z}\rangle$, and the $R_{\text {aa }} 2 \mathrm{D}$ roughness parameter (defined as $\mathrm{R}_{\mathrm{aa}} \equiv \frac{1}{\mathrm{~A}} \iint_{\mathrm{A}}|\mathrm{z}(\mathrm{x}, \mathrm{y})-\overline{\mathrm{z}}| \mathrm{dA}$ ) are shown for different values of EOD in both materials.
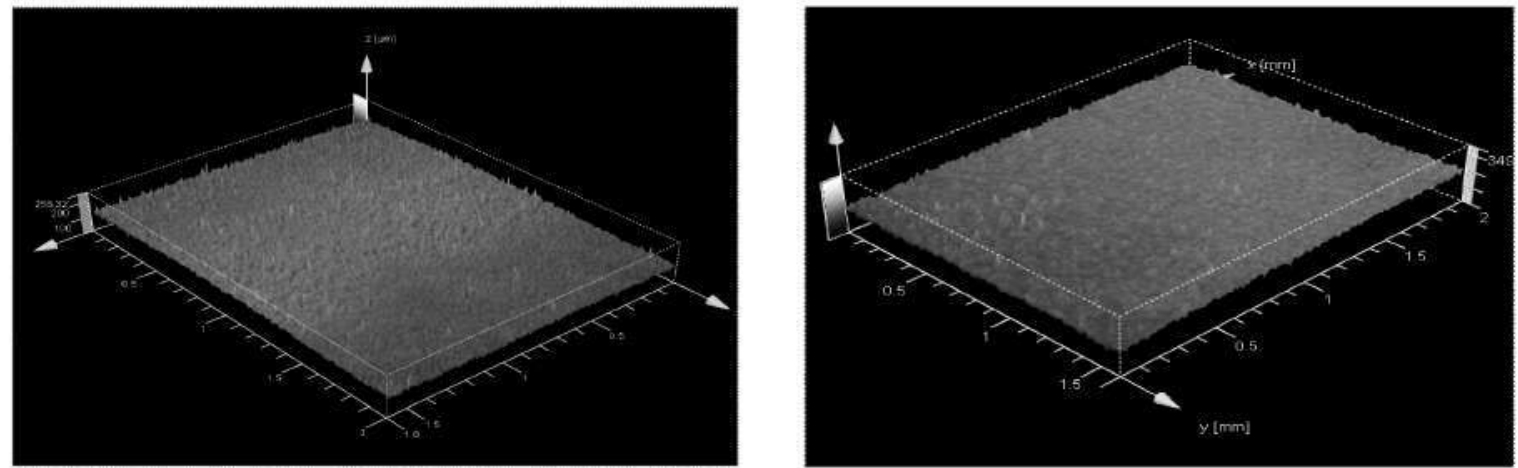

Figure 4: Topographic Laser Confocal Microscope images showing the changes in surface roughness induced by the LSP technique in near-optimum conditions for Al2024T351 (left) and Ti6A14V (right).

\begin{tabular}{|c|c|c|c|c|c|}
\hline Treatment EOD & $\begin{array}{c}\text { No } \\
\text { treatment }\end{array}$ & $\begin{array}{c}\mathbf{9 0 0} \\
\text { pulses } / \mathbf{c m}^{2}\end{array}$ & $\begin{array}{c}\mathbf{1 6 0 0} \\
\text { pulses } / \mathbf{c m}^{2}\end{array}$ & $\begin{array}{c}\mathbf{2 5 0 0} \\
\text { pulses } / \mathbf{c m}^{2}\end{array}$ & $\begin{array}{c}\mathbf{5 0 0 0} \\
\text { pulses } / \mathbf{c m}^{2}\end{array}$ \\
\hline$<\Delta \mathbf{z}>\mathbf{A l 2 0 2 4 - T 3 5 1}(\mu \mathrm{m})$ & ---- & 10.30 & 20.00 & 26.82 & \\
\hline $\mathbf{R}_{\mathrm{aa}}(\mu \mathrm{m}) \mathbf{A l 2 0 2 4 - T 3 5 1}(\mu \mathrm{m})$ & 7.96 & 5.23 & 4.82 & 4.96 & \\
\hline$<\Delta \mathbf{z}>$ Ti6Al4V $(\mu \mathrm{m})$ & --- & 2.81 & 7.40 & & 5.80 \\
\hline $\mathbf{R}_{\mathrm{aa}}(\mu \mathrm{m})$ Ti6Al4V $(\mu \mathrm{m})$ & 9.98 & 3.62 & 3.87 & & 3.87 \\
\hline
\end{tabular}

Table 1. Summary of surface compactation results, $\langle\Delta \mathbf{z}\rangle$, and $2 \mathrm{D}$ surface roughness parameter $\mathrm{R}_{\mathrm{aa}}$ for different values of EOD in A12024-T351 and Ti6Al4V.

On the basis of these results, the idea can be extracted of a rather significant surface smoothing provided by the LSP treatment, a key feature in view of the fatigue fretting and corrosion resistance of specimens treated by this technique, although the effect cannot, in general, be so directly controlled as the residual stresses fields by means of increasing treatment EOD's.

\section{Wear resistance}

The just described results on surface roughness lowering by LSP have also a clear effect on the abrasive wear resistance of the treated specimens, although other possible surface transformation mechanisms (namely surface oxidation) finally determine the wear resistance of samples. In this case, materials able to experiment a physic-chemical transformation (carburization, nitriding, oxidation, etc.) can exhibit a differentiated behaviour concerning this property beyond the mechanical effects purely attributable to the LSP treatment.

As a clear example in this line, the comparative case of the two alloys considered in this paper (Al2024-T351 and Ti6Al4V) shows the difference between the wear behaviour of two crystalline 
phases developed by the LSP treatment in both materials with very different surface hardness properties, namely $\mathrm{Al}_{2} \mathrm{O}_{3}$ and $\mathrm{TiO}_{2}$, both appeared as a consequence of the surface heating resulting from the plasma generated by the incident laser beam. Although it has been shown by the authors that the effect of this plasma heating is not of a critical importance from the point of view of the residual stresses field obtained in the treated material after a certain depth [16], the effect of such heating seems to be important in what respects to hardness and wear properties.

In Fig. 5, the results of the wear resistance of the reference alloys for a $20 \mathrm{~N}$ load according to the ASTM G99-04 Standard are shown. It is clearly observed that, despite the slight variations induced in the friction coefficient as a consequence of the LSP treatment (responsible for the $2^{\text {nd }}$ order effects observed in the graphs, in general favourable to higher EOP LSP treatments), the appearance of a "hard" phase in A12024-T351 alloy following LSP treatment (that can be very clearly observed in SEM microstructure and EDX elemental analysis and is absent in the case of Ti6Al4V) provides a physic-chemical mechanism for enhanced wear resistance.
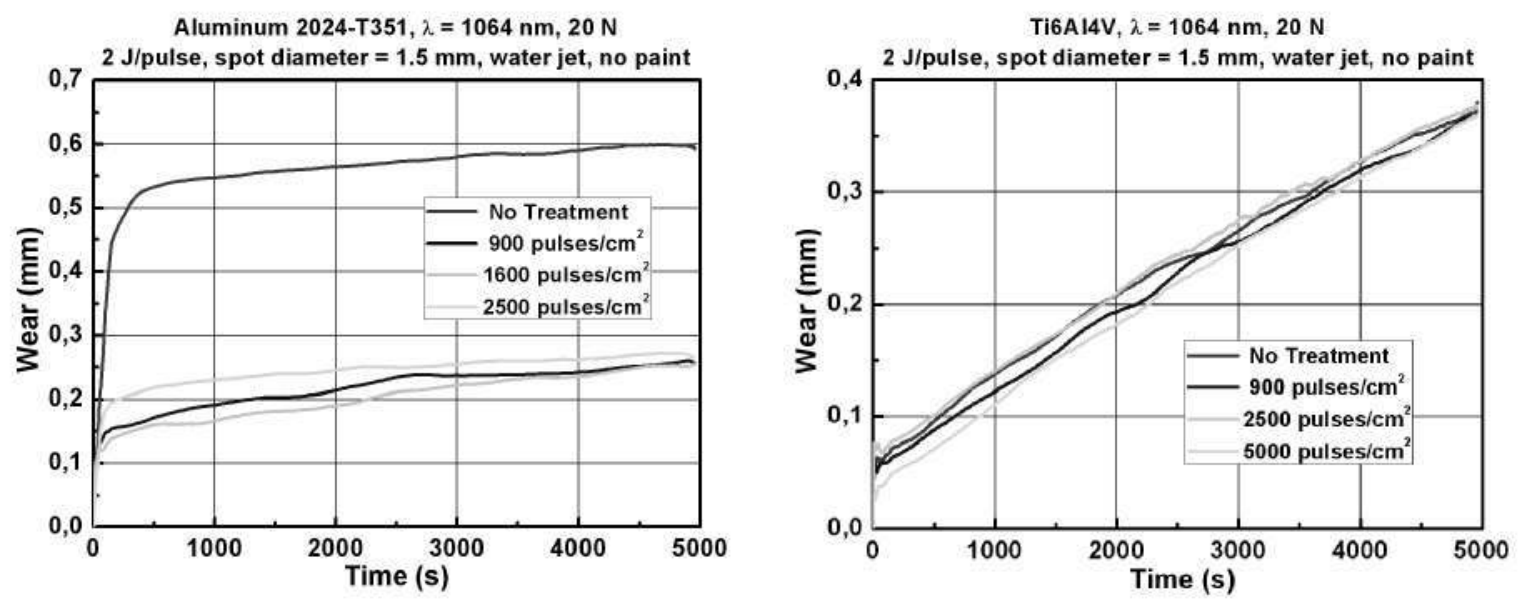

Figure 5. Wear resistance results according to ASTM G99-04 Standard of Al2024-T351 and Ti6Al4V specimens treated by LSP with different EOD's

\section{Discussion}

A summary of surface effects induced by the LSP technique in two metallic alloys typical of the aerospace industry has been presented, a special focus on surface morphology and wear behaviour having been selected for this paper.

Considering its primary interest for a broad range of mechanical properties susceptible to be enhanced by the LSP technique, a sample of the residual stresses depth profiles obtained in the considered materials for different treatment intensities (accounted for through the EOD parameter). The observation of these results clearly leads to the consideration of the LSP technique as really effective and controllable within wide limits for the induction of engineered residual stresses fields in critical components through modification of specific mechanical and chemical material properties, especially mechanical fatigue and corrosion resistance. The effect has been widely demonstrated by the authors in simple geometries [11-14] and, with the aid of the developed calculational tools [9-10], can be readily applied to arbitrary component geometries.

Considering other important surface properties, an analysis has been presented of the effect of LSP on surface roughness, a relevant parameter conditioning the appearance of mechanical or corrosion failure at the surface level. In this case, LSP has been clearly shown to exert a positive effect on the surface evenness, presumably beneficial concerning specifically fatigue fretting and pitting corrosion.

Finally, the analysis of wear resistance of specimens of both materials treated by the LSP technique has been reported, the important fact having being found of the significant influence of the ambient processing conditions on the final mechanical performance of the treated surface. 
Concretely, the relatively important influence of possible oxidation or other physic-chemical surface modification mechanisms in the case of alloys presenting the appropriate elemental composition has been reported.

This fact has to be considered of a high relevance in view of its inherent difficulty to be adequately controlled. Both the control of atmospheric treatment conditions and the dynamics of the laser produced plasma near the material surface are key issues deserving a further detailed evaluation.

\section{Acknowledgements}

Work partly supported by MEC/MCINN (Spain; Projects DPI2005-09152-C02-01 and MAT200802704/MAT) and EADS-CASA (Spain).

\section{References}

[1] B.P. Fairand et al. J. Appl. Phys., $\underline{43}$ (1997), 3893-3896.

[2] L.C. Yang: J. Appl. Phys., 45 (1974), 2601-2607.

[3] B.P. Fairand, A.H. Clauer: J. Appl, Phys., $\underline{50}$ (1979), 1497-1502.

[4] Y. Sano et al:: "Underwater Laser Processing to Improve Residual Stress on Metal Surface". In: Proceedings of the 6th International Welding Symposium of Japan Welding Society, 1996, Nagoya, Japan, 501-506.

[5] C.B. Dane: "High Power Laser for Peening of Metals Enabling Production Technology". In: Lawrence Livermore National Laboratory, Report UCRL-JC-131104-Rev 1 (1998).

[6] Y. Sano et al.: Nucl. Inst. Meth. Phys. Res. B, 121 (1997), 432-436.

[7] L. Berthe et al.: J. Appl. Phys., $\underline{85}$ (1999), 7552-7555.

[8] K. Akita et al.: "Compressive Residual Stress Evolution Process by Laser Peening". In Materials Science Forum Vols. 490-491 (2005), 370-375.

[9] J.L. Ocaña et al.: "Predictive assessment and experimental characterization of the influence of irradiation parameters on surface deformation and residual stresses in laser shock processed metallic alloys". In: High-Power Laser Ablation V, Phipps C.R., Ed.. SPIE Vol. $\underline{5488}$ (2004) 642-653.

[10] J.L. Ocaña et al.: Appl. Surf. Sci,, 238 (2004), 242-248.

[11] C. Rubio-González et al.: Mat. Sci. Eng. A, 386 (2004) 291-295.

[12] J.L. Ocaña et al.: "Laser Shock Processing as a Method for Surface Properties Modification of Metallic Materials". In: Shot Peening and other Mechanical Surface Treatments. V. Schulze,

A. Niku-Lari, Eds. I.I.T.T. Paris (2005) 466-471.

[13] U. Sánchez-Santana et al.: Wear, 260 (2006) 847-854.

[14] C. Rubio-González et al:: Appl. Surf. Sci., 252 (2006) 6201-6205.

[15] J. Grum et al.: Mat. Sci. Forum Vols. 537-538 (2007), 655-662.

[16] J.L. Ocaña et al.: Mat. Sci. Forum Vols. 539-543 (2007), 1116-1121.

[17] M. Morales et al.: Surface \& Coatings Technology 202 (2008) 2257-2262.

[18] ASTM International 2002, v.03.01: "E837-01 Standard Test Method for Determining Residual Stresses by the Hole-Drilling Strain Gage Method".

[19] ASTM International 2004: "ASTM G 99-04 Standard Test Method for Wear Testing with a Pin on Disk Apparatus".

[20] T. Nicholas: Int. J. Fatigue, 21(S) (1999), S221-S231.

[21] A.L. Hutson, T. Nicholas: Tribol. Int. 36 (2003), 133-143. 